\title{
An Unusual Masticator Space Infection Initially Presenting as Temporomandibular Joint Dislocation: A Case Report
}

\section{Nan En Ho ${ }^{1}$ and Hao-Hueng Chang ${ }^{1,2^{*}}$}

${ }^{1}$ Department of Dentistry, National Taiwan University Hospital, College of Medicine, National Taiwan University, Taipei, Taiwan

${ }^{2}$ Graduate Institute of Clinical Dentistry and Department of Dentistry, School of Dentistry, National Taiwan University, Taipei, Taiwan

*Corresponding authors: Hao-Hueng Chang, School of Dentistry, National Taiwan University Hospital and Medical College, National Taiwan University, Number 1, Chang-Te Street, Taipei City 100, Taiwan, Tel: +88623123456 (ext: 66847 or 66847); Fax: 886-23831346; E-mail: changhh@ntu.edu.tw

Recieved Date: April 24, 2017; Accepted Date: May 03, 2017; Published Date: May 08, 2017

Copyright: $\odot 2017 \mathrm{Ho} \mathrm{NE}$, et al. This is an open-access article distributed under the terms of the Creative Commons Attribution License, which permits unrestricted use, distribution, and reproduction in any medium, provided the original author and source are credited.

\begin{abstract}
Masticator space infection can easily be misdiagnosed as temporomandibular joint (TMJ) disorder. However, the association between masticator space infection and TMJ dislocation has not been studied. The present study describes a case of masticator space infection that initially presented as TMJ dislocation.

A 63-year-old woman complained of dislocation and pain on the right TMJ for 3 weeks. Physical examination revealed right TMJ dislocation, which was confirmed by computed tomography (CT). Bimanual reduction of the dislocated TMJ was performed under general anesthesia with intermaxillary fixation. However, progressive right facial swelling was noted after the closed reduction of the right TMJ. Magnetic Resonance Imaging (MRI) revealed marked soft tissue swelling and mass forming-like change in the periarticular region of the right TMJ. Moreover, a necrotic area of the masseter and buccinator muscles was noted, which indicated dislocation-induced injury or hemorrhage. However, neoplasm arising from the joint space could not be ruled out. CT-guided biopsy was then performed, which showed chronic inflammation and necrotic tissue in the right masticator space, thus excluding the possibility of neoplasm. Extraoral Incision and Drainage (I\&D) was then performed, and the final pathologic diagnosis coincident with cellulitis involved the masticator space and parotid spaces. After I\&D and surgical debridement, the patient recovered satisfactorily.
\end{abstract}

Although rare, we must consider the possibility of deep space infection when diagnosing patients presenting TMJ dislocation for their adequate and prompt management.

Keywords: Masticator space infection; Temporomandibular joint dislocation

\section{Introduction}

The temporomandibular joint (TMJ), which assists mandible movement and lower jaw opening, comprises the glenoid fossa of the temporal bone, the condylar head of the mandible, the articular disk, and several ligaments and associated muscles. In TMJ dislocation, the condylar head is positioned anterior and superior to the articular eminence without self-reduction ability [1]. Furthermore, the dislocation can be classified as acute, chronic protracted or chronic recurrent [2].

The possible etiology is excessive mouth opening caused either by self-induced or iatrogenic forces or predisposing factors such as epilepsy, Ehlers-Danlos syndrome, Marfan's syndrome and altered structural components [2]. Conservative reduction of the TMJ is the most common management strategy. For chronic recurrent dislocation, the treatment focuses on restricting condylar movement or eliminating the self-reduction obstacle [1-3].

The masticator space, a collective term for the superficial and deep temporal, submasseteric, and pterygomandibular spaces, is confined by the masticator muscles including the temporalis, masseter, lateral, and medial pterygoid muscles [4]. Masticator space infection (MSI), which is mostly caused by the spread of odontogenic infection, may present as TMJ pain, masticator muscle tenderness, or trismus. Without obvious dental infection, MSI can easily be misdiagnosed as TMJ disorder (TMD) $[4,5]$.

In this study, we report a case of MSI that initially presented as TMJ dislocation.

\section{Case Report}

A 63-year-old woman visited the emergency room (ER) for dislocation and pain on the right TMJ for 3 weeks. She reported that her occlusion changed gradually and complained of severe malocclusion and poor chewing function. Her vital signs were stable, and no fever was noted. Physical examination revealed right TMJ dislocation without local heat but mild swelling. Malocclusion with an open bite and limited mouth opening of approximately $20 \mathrm{~mm}$ were also noted.

The panoramic radiograph showed right TMJ dislocation and no evidence of odontogenic pathology (Figure 1). Laboratory studies showed a white blood cell count (WBC) of $12.24 \mathrm{~K} / \mu \mathrm{l}$. Computed tomography (CT) confirmed the diagnosis of TMJ dislocation with perifocal fat stranding near the right masticator space and signal enhancement near the right TMJ and the right pterygoid muscle (Figure 2A and 2B). 
Citation: Ho NE, Chang HH (2017) An Unusual Masticator Space Infection Initially Presenting as Temporomandibular Joint Dislocation: A Case Report. Dentistry 7: 434. doi:10.4172/2161-1122.1000434

Page 2 of 4

Bimanual reduction of the dislocated TMJ was failed in the ER setting, we thus performed intermaxillary fixation following closed reduction of right TMJ under general anesthesia. However, progressive right facial swelling was evident following closed reduction. Empirical antibiotics were administered.

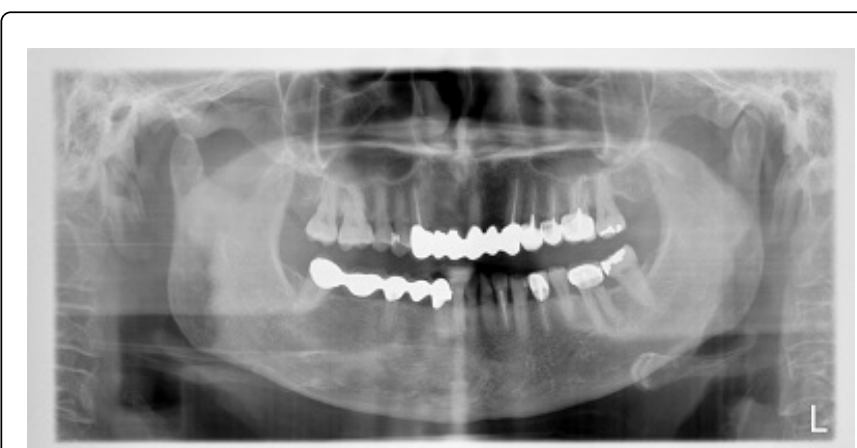

Figure 1: Panoramic radiograph showing right temporomandibular joint dislocation and no evidence of odontogenic pathology.

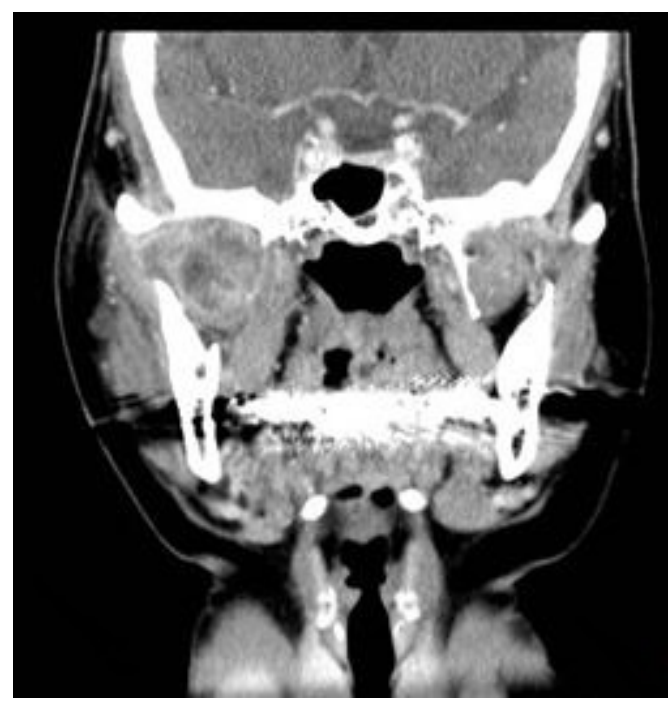

Figure 2(A): Computed Tomography (CT) image before Temporomandibular Joint (TMJ) reduction: right TMJ dislocation with perifocal fat stranding near the right masticator space and signal enhancement near the right TMJ and the right pterygoid muscle.

Magnetic resonance imaging (MRI) revealed marked soft tissue swelling and mass forming-like change in the periarticular region of the right TMJ. Moreover, a necrotic area of the masseter and buccinator muscles was noted, indicating infection, hemorrhage, or neoplasm (Figure 3). CT-guided biopsy demonstrated chronic inflammation and necrotic tissue in the right masticator space, thus excluding the possibility of neoplasm arising from the joint space (Figure 2C).

Extraoral incision and drainage (I\&D) was then performed, and the final pathologic diagnosis coincident with cellulitis involved masticator space and parotid spaces. After I\&D and surgical debridement, the swelling subsided, and the patient's maximum mouth opening improved to $30^{+} \mathrm{mm}$.

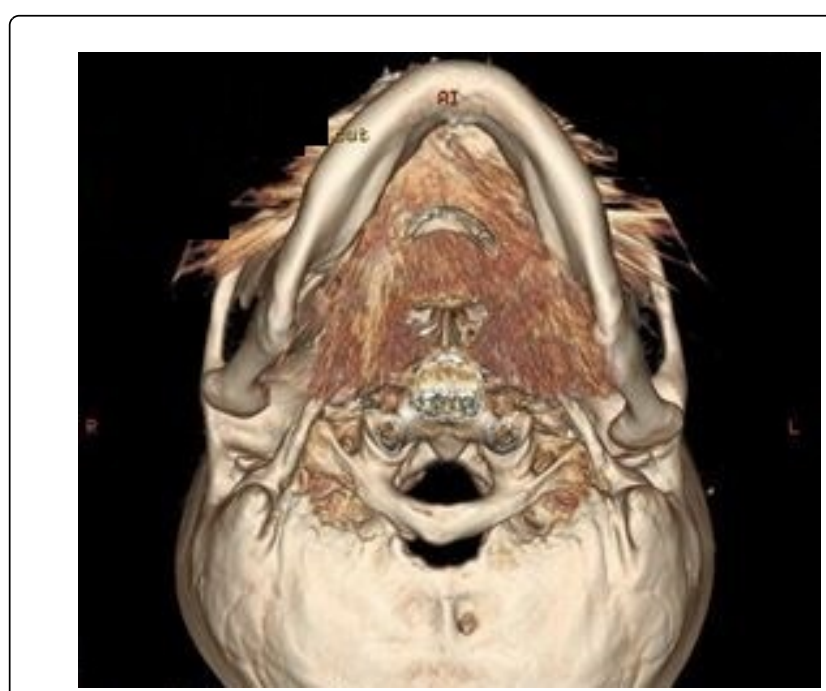

Figure 2(B): CT image before TMJ reduction with 3D reconstruction.

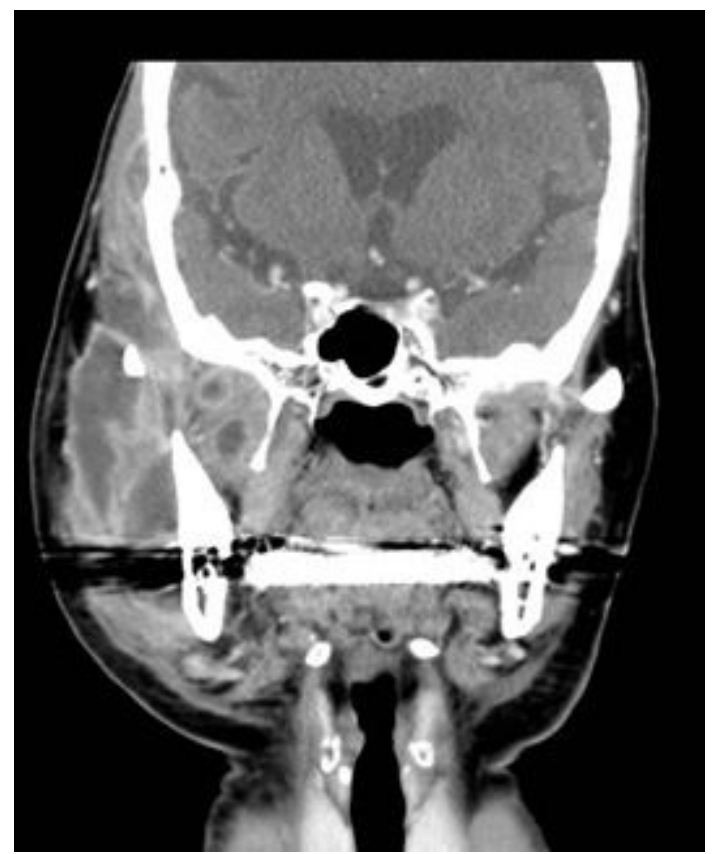

Figure 2(C): CT image after TMJ reduction: abscess spread extensively in the masticator and buccal space.

\section{Discussion}

TMJ dislocation is usually a sudden episode that results from excessive mouth opening. Excessive mouth opening may be caused by self-induced forces such as yawning, laughing, vomiting, and seizure or iatrogenic forces such as dental treatment, endotracheal intubation and laryngoscopy [1]. 


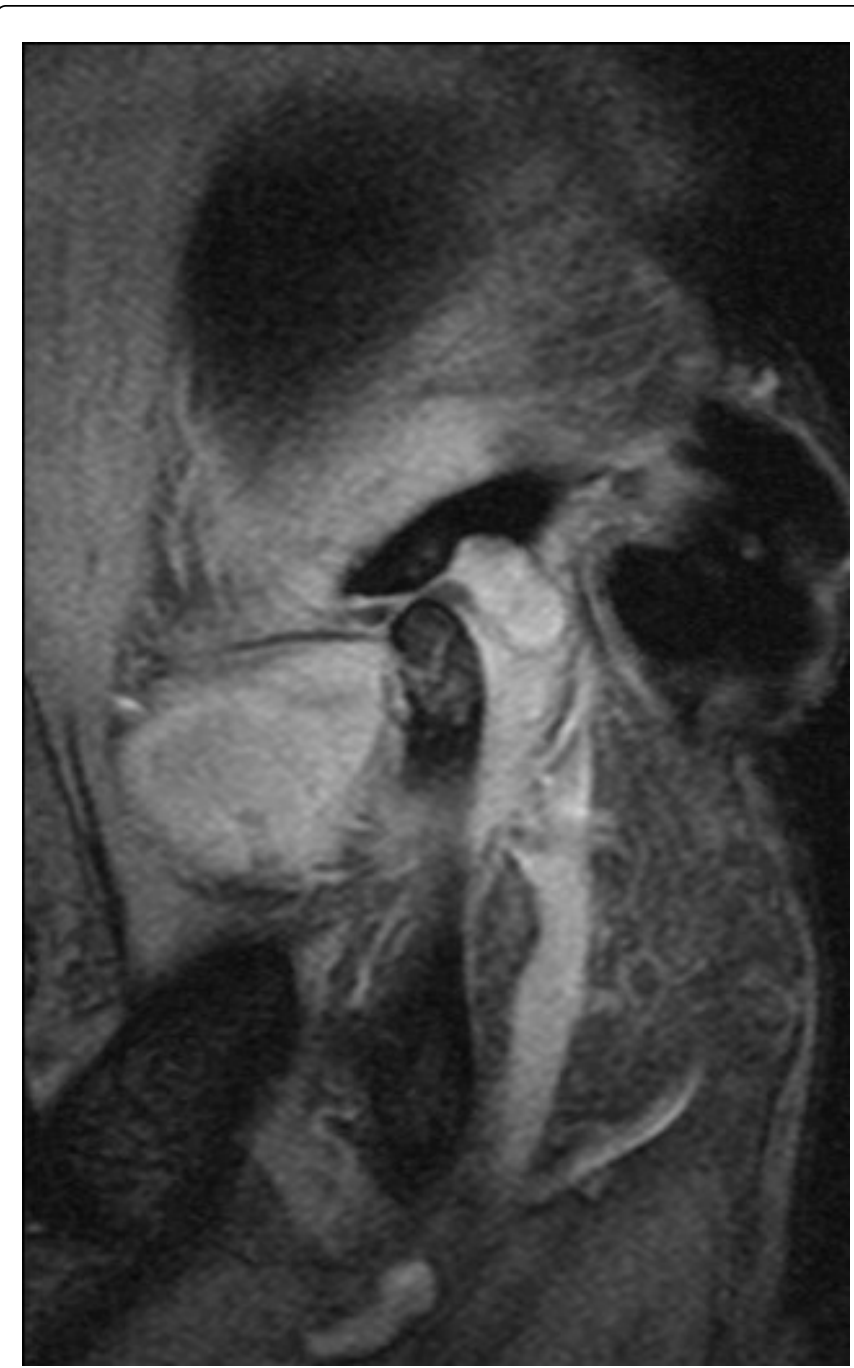

Figure 3: Magnetic Resonance Imaging (MRI) revealed right Temporomandibular Joint (TMJ) dislocation, limited range of movement, marked soft tissue swelling, and mass forming-like change with enhancement in the periarticular region of the right TMJ, as well as a necrotic area of the masseter and buccinator muscles.

Moreover, predisposing factors such as epilepsy, Ehlers-Danlos syndrome, Marfan's syndrome and certain antipsychotic medications may cause TMJ dislocation. Imbalance in the neuromuscular function and structural deficits may also lead to TMJ dislocation. If the dislocation remains untreated, the condition may become chronic or recurrent, and acute dislocation may be transformed into chronic protracted or chronic recurrent dislocation $[1,2]$. In our patient, although imaging studies confirmed TMJ dislocation, the patient did not have any history of sudden onset but exhibited gradual deterioration. Therefore, since the first visit to the ER, neoplasm was included in our differential diagnosis. Another possibility was that prolonged unnoticed dislocation may induce muscle spasm and inflammatory changes, which caused the occlusion changed gradually.

According to the consensus on TMJ dislocation treatment, the initial treatment should include conservative methods, and surgical methods should only be used if conservative treatment fails. Conservative methods refer to pain relief and bimanual reduction of the TMJ under local or general anesthesia. For chronic protracted dislocation, IMF with elastic bands may be considered. Surgical methods can be classified into strategies restricting TMJ movement or removing a mechanical obstacle to achieve easy reduction of TMJ. Strategies restricting TMJ movement include injecting sclerosing agents or blood to enhance fibrosis, or using autogenous bone graft or implants to augment the articular eminence. In this study, we performed bimanual reduction under local anesthesia, but the severe pain could not be tolerated by the patient. Therefore, we reduced the joint under general anesthesia and performed IMF owing to prolonged dislocation. However, for cases with a possible diagnosis of neoplasm and further clarified MSI, the common management strategy of joint reduction for dislocation may not be appropriate.

The symptoms of MSI mimic TMD, particularly when there is no odontogenic infection, and can easily be misdiagnosed. Trismus, TMJ pain, masticator muscle tenderness are common presentations for both diseases. Hasegawa et al presented two cases of MSI that were initially misdiagnosed as TMD. After accurate interpretation and prompt treatment, the patients satisfactorily recovered and were discharged [5]. Although odontogenic infection is the most common etiology for MSI, other possible etiologies are bacterial or viral pharyngitis, retropharyngeal abscess, peritonsillar abscess, acute otitis media, mastoiditis, parotitis, submandibular sialadenitis and lymphadenopathy, which should be included in the differential diagnosis [6]. In addition, some unusual etiologies exist. Ko et al. reported a case with abscess formation in the masticator space after acupressure massage, which was initially diagnosed as TMD. After CT imaging and needle aspiration, the treatment focused on MSI and the patient's symptoms improved [4]. Matsumura et al. also presented a case with peritemporomandibular abscess as a complication of acupuncture [7]. Although MSI may be misdiagnosed as TMD, currently, no reports or evidence indicates that TMJ dislocation is associated with MSI.

To clarify the association between TMJ dislocation and MSI, we made the most reasonable assumption. We hypothesized that MSI initially presented with some unknown etiology. The inflammatory reaction further led to lateral pterygoid muscle spasm and the local swelling on the retrodiscal area resulted in TMJ anterior displacement. The two effects combined to cause TMJ dislocation, and the condition worsened as the infection persisted and progressed. The persistence of the dislocated joint further evolved inflammatory changes and muscle spasm, creating a vicious cycle. Under this assumption, we should consider the rare cause of MSI when diagnosing patients presenting TMJ dislocation.

\section{Conclusion}

Based on this case report, TMJ dislocation may be caused by MSI, although infrequently. We should consider this unusual possibility when diagnosing patients complaining of a dislocated TMJ, particularly those with an aberrant history of disease progression.

\section{References}

1. Sharma NK, Singh AK, Pandey A, Verma VS (2015) Temporomandibular joint dislocation. Natl J Maxillofac Surg 6: 16-20.

2. Akinbami BO (2011) Evaluation of the mechanism and principles of management of temporomandibular joint dislocation. Systematic review 
Citation: Ho NE, Chang HH (2017) An Unusual Masticator Space Infection Initially Presenting as Temporomandibular Joint Dislocation: A Case Report. Dentistry 7: 434. doi:10.4172/2161-1122.1000434

Page 4 of 4

of literature and a proposed new classification of temporomandibular joint dislocation. Head Face Med 7: 10.

3. Vasconcelos BC, Porto GG (2009) Treatment of chronic mandibular dislocations: a comparison between eminectomy and miniplates. J Oral Maxillofac Surg 67: 2599-2604.

4. Ko IC, Yoon KH, Park KS, Cheong JK, Bae JH, et al. (2015) An unusual abscess formation in the masticator space after acupressure massage: a case report. J Korean Assoc Oral Maxillofac Surg 41: 52-56.

5. Hasegawa T, Shibuya Y, Kuroki S, Takeuchi J, Yokoo S, et al. (2008) Two cases of masticator space abscess initially diagnosed as temporomandibular joint disorder. Kobe J Med Sci 54: 163-168.
6. Gayle EA, Young SM, Kenna MC, Naughton CD (2013) Septic arthritis of the temporomandibular joint: case reports and review of the literature. J Emerg Med 45: 674-678.

7. Matsumura Y, Inui MT, Agawa T (1998) Peritemporomandibular abscess as a complication of acupuncture: a case report. J Oral Maxillofac Surg 56: 495-496. 